\title{
Crystal Structures of Human FIH-1 in Complex with Quinol Family Inhibitors
}

Hyunjin Moon, Sojung Han, Hyunsung Park, and Jungwoo Choe*

Department of Life Science, University of Seoul, Seoul 130-743, Korea

In the article by Hyunjin Moon et al. that appears in the journal (Mol. Cells 29, 471-474, May 31, 2010), AUTHORS' AFFILIATIONS should be revised as written below.

Hyunjin Moon ${ }^{1}$, Sojung Han ${ }^{1}$, Hyunsung Park, and Jungwoo Choe*

Department of Life Science, University of Seoul, Seoul 130-743, Korea, ${ }^{1}$ These authors contributed equally to this work. 